

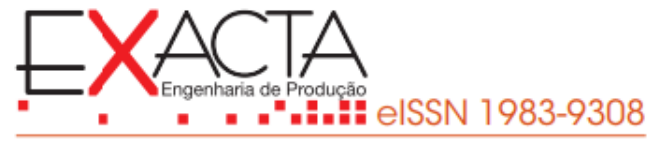

\title{
INDÚSTRIA 4.0: IMPACTOS DAS NOVAS TECNOLOGIAS NO GERENCIAMENTO DE PROJETOS
}

\author{
INDUSTRY 4.0: IMPACTS OF NEW TECHNOLOGIES ON PROJECT MANAGEMENT
}

\author{
D Igor Bernardino Borges ${ }^{1}$ \\ D Josivan Leite Alves ${ }^{2}$ \\ iD Leonardo Kennedy Alves de Lima ${ }^{3}$ \\ iD Jeniffer de $\mathrm{Nadae}^{4}$
}

${ }^{1}$ Mestrando em Engenharia Civil Universidade Federal de Pernambuco - UFPE. Recife, Pernambuco - Brasil. igor.bernardino@ufpe.br

2 Mestrando em Engenharia de Produção Universidade de São Paulo - USP. São Paulo, São Paulo- Brasil. josivan.leite@usp.br

\section{Engenheiro Civil} Universidade Federal do Cariri- UFCA. Juazeiro do Norte, Ceará - Brasil. leonardokennedyalvesdelima@gmail.com

4 PHD em Engenharia de Produção Universidade Federal de Itajubá - UNIFEI. Itajubá, Minas Gerais - Brasil. jeniffer.nadae@unifei.edu.br

Recebido em: 26 jul. 2020 Aprovado em: 25 out. 2020
Resumo: A quarta revolução industrial, Industria 4.0, carrega consigo o legado de assegurar a evolução humana, de forma a dispor ferramentas que podem auxiliar os tomadores de decisão em diversos campos, como: maior controle, desempenho e produtividade em todo o ciclo de vida de um projeto. Desta forma, o objetivo deste trabalho é analisar a relação entre a indústria 4.0 e a gestão de projetos por meio de análises de literatura, identificando os principais impactos das novas tecnologias no gerenciamento de projetos atual. Para a metodologia utilizou-se de um estudo bibliométrico e uma análise de conteúdo, utilizando a base de dados da Scopus. Através das análises foi possível classificar os documentos em seis pilares principais da Indústria 4.0 em relação ao gerenciamento de projetos: internet das coisas, sistemas cyber-físicos, big data, automação, inteligência artificial e computação em nuvem, assim como, observar como as novas tecnologias desenvolvemse quanto ao gerenciamento de projetos. Além disso, nos dois últimos anos, notou-se um crescimento na quantidade de publicações sobre o tema revelando oportunidades para futuras pesquisas. Ademais, pode-se perceber que o setor privado promove o desenvolvimento de diferentes pesquisas de gerenciamento, como também, melhoramento dos sistemas já existentes com a implementação de computação em nuvem e internet das coisas, contribuindo assim, para a teoria sobre o tema. Percebe-se que os desafios para a implementação da Indústria 4.0 variam de acordo com cada país, devendo-se levar em consideração: o estágio de desenvolvimento das bases industriais, origem dos principais investidores e a existência de políticas públicas que incentivem o desenvolvimento de pesquisas sobre o tema, desta forma este estudo contribui para a prática organizacional. quanto a comunidade em geral.

Palavras-chave: Indústria 4.0. Gerenciamento de projetos. Novas tecnologias. Análise bibliométrica.

Abstract: The fourth industrial revolution, Industry 4.0, carries with it the legacy of ensuring human evolution, in order to provide tools that can help decision makers in various fields, such as: greater control, performance and productivity throughout the complete life cycle of a project. Thus, this work aim's is to analyze the relationship between industry 4.0 and project management through literature analysis, identifying the main impacts of new technologies on current project management. For the methodology, a bibliometric study and a content analysis were used, using the Scopus database. Through the analysis it was possible to classify the documents in six main pillars of Industry 4.0 regarding project management: Internet of things, cyber-physical systems, big data, automation, artificial intelligence and cloud computing, as well as, observe how the new technologies are developed regarding project management. Also, in the last two years, there has been an increase in the number of publications on the subject, revealing opportunities for future research. Furthermore, it can be seen that the private sector promotes the development of different management researches, as well as improving existing systems with the implementation of cloud computing and the internet of things, thus contributing to the theory on the subject. It is noticed that the challenges for the implementation of Industry 4.0 vary according to each country, taking into account: the stage of development of the industrial bases, the origin of the main investors, and the existence of public policies that encourage the development of research on the topic, in this way this study contributes to the organizational practice.

Keywords: Industry 4.0. Project management. New technologies, Bibliometric analysis. 


\section{Introdução}

A evolução da humanidade resulta na criação de novos mecanismos produtivos de acordo com as ferramentas disponíveis. As três primeiras revoluções industriais configuram-se pelo surgimento ferramentas que transformaram os processos de produção, como: as máquinas a vapor, uso do aço, motor elétrico e sistemas computacionais. Neste momento, a revolução industrial corresponde a de número quatro, também conhecida como indústria 4.0 onde a internet das coisas, inteligência artificial, sistemas integrados e comunicação dentro da indústria representam sua marca (Anderl, 2015).

Segundo Schmitt (2019) o aumento na complexidade dos processos corresponde a um indicador de progresso para humanidade, sendo as mudanças na tecnologia consideradas a " 4 a revolução industrial". De modo a adaptar-se as crescentes expectativas do mercado, necessidades individualizadas e específicas de cada cliente, o ciclo de vida dos produtos precisa de maior controle e organização (Geissbauer et al., 2014).

Desta forma, a indústria 4.0 abrange instrumentos capazes de assegurar a evolução humana, dentre eles pode-se citar os seguintes benefícios: Estratégicos onde estão inseridos os três pilares da sustentabilidade, otimização do lucro e maior capacidade de integração ao mercado; táticos com os sistemas cyber físicos, internet das coisas e tecnologias na cadeia produtiva; e operacionais por meio do aumento da produtividade, conectividade e análises em tempo real (Senai, 2019).

Para Blanco e Oliveira (2018) as formas de trabalho mudam à medida que as tecnologias avançam, de forma que a Indústria 4.0 já está presente na elaboração e gestão de projetos, bem como em linhas de pesquisa em a nível mundial. Ou seja, um novo paradigma da manufatura está instaurado, sendo este marcado pela produção inteligente e máquinas interconectadas ao longo de toda a cadeia de valor (Qin; Liu; Grosvenor, 2016), que alteram significativamente o gerenciamento de projetos (Cavalcanti et al., 2018).

A Indústria 4.0 abrange todos os setores a exemplo da construção civil em que ferramentas que utilizam a tecnologia Building Information Modeling - BIM (ou Modelagem de Informações da Construção) conseguem formular todos os projetos de forma integrada, dispondo de todas as informações de cada etapa da obra (Cavalcanti et al., 2018).

É válido ressaltar que o conceito de projetos é entendido como a realização esforços temporários junto a um ciclo de vida específico de modo a atingir um objetivo, seja por mobilização de recursos humanos e financeiros, seja por recursos materiais (Schelini; Martens; Piscopo, 2017). Sendo estes realizados com o intuído de produzir entregas: produto, serviço, resultado ou combinação de produtos, serviços ou resultados (PMI, 2018).

Dessa forma, para o alcance dos objetivos de cada projeto é necessário que haja um bom gerenciamento do mesmo, o qual deve contar com aplicação e integração adequadas dos processos de 
gerenciamento (PMI, 2018). Gerenciamento de projetos, segundo Vargas (2005) corresponde a um processo estruturado e lógico de forma a tratar de eventos novos, complexos e dinâmicos.

Ao mesmo tempo, existem ferramentas e técnicas que assessoram na condução de escopo de um projeto como: ferramentas de gerenciamento, controle de qualidade, custos e riscos (PMI, 2018). Tais mecanismos estabelecem habilidades, iniciativas e estratégias para alcançar um bom resultado do projeto, como também, analisam às áreas de conhecimentos de cada um dos envolvidos na gestão (Kameiya; Romeiro; Kniess, 2017).

Sendo assim, a justificativa de realizar esta pesquisa fundamenta-se em explorar como as produções internacionais relacionam o gerenciamento de projetos com a utilização de tecnologias provindas da quarta revolução industrial. Tendo o objetivo pautado em analisar a relação entre a indústria 4.0 e a gestão de projetos por meio de análise bibliométrica e de conteúdo, identificando os impactos das novas tecnologias no gerenciamento de projetos atual. O método de pesquisa utilizado foi compreendido na realização de análise bibliométrica e de conteúdo na base de dados Scopus.

Para tal este segue estruturado da seguinte forma: introdução sobre o tema abordado, fundamentação teórica expondo a literatura a respeito da temática abordada, metodologia e considerações finais.

\section{Referencial Teórico}

A ideia de indústria 4.0 surgiu na Alemanha como "Industrie 4.0" por meio da feira Hannover Messe edição de 2011 (Kagermann; Lukas; Wahlster, 2011). Sendo também disseminada em publicações sobre políticas e tecnologias em todo o mundo, em 2015 a comissão europeia criou o Horizon 2020 com o intuito de promover a expansão da indústria 4.0 (Davies, 2015).

O surgimento da internet corresponde a um dos principais pilares que sustentam a quarta revolução industrial pois esta permitiu uma comunicação mais rápida e eficiente tanto a curtas, quanto a longas distâncias entre pessoas e equipamentos. A expansão das tecnologias representa outra base uma vez que proporciona desde a criação de sensores, ao barateamento de equipamentos. (Cavalcanti et al., 2018). A figura 1 corresponde as revoluções industriais e suas respectivas marcas. 


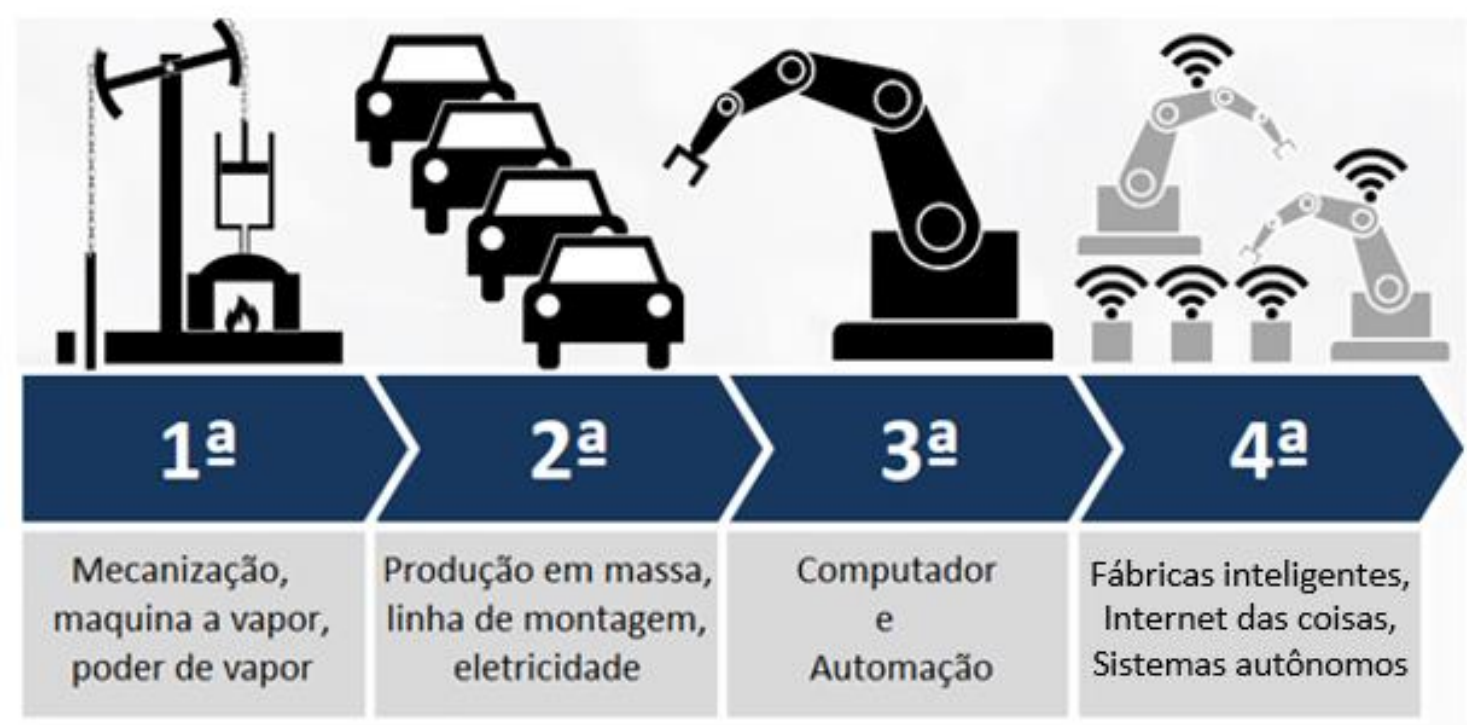

Fonte: Adaptado Jamison (2019).

Paralelamente, a produção inteligente surge como um modelo produtivo com melhor controle na concepção, produção, gestão e integração (Zhong et al., 2017). Por meio desta, novos produtos são desenvolvidos com eficiência e qualidade utilizando materiais avançados, máquinas inteligentes e análises de dados automáticas capazes se adaptar a diversos cenários (Davis et al., 2012).

Segundo Kolberg et al. (2015) a ideia de unir tecnologias de automação com produção surgiu nos anos 90 com um computador integrado a produção. A implementação de máquinas na indústria resulta em otimização e por consequência aumento no seu lucro, a exemplo pode-se citar a implementação de sensores em uma mina de ouro na África que resultaram em uma economia de 3,7\% de seus rendimentos, cerca de 20 milhões de dólares por ano (Marr, 2018).

Em pesquisa divulgada pelo Ministério da Indústria, Comércio e Serviços do Brasil (2019), estima-se que a migração da indústria para o conceito 4.0 que segundo Ribeiro (2017 p. 8) "teve origem em um projeto estratégico de alta tecnologia criado e desenvolvido pelo governo alemão, cujo objetivo era a promoção da informatização da indústria" somente em território brasileiro economizará cerca de 73 bilhões de reais por ano graças a ganhos de eficiência, redução de custos de manutenção e consumo de energia.

Em síntese Cakmakci (2019) define que existem três particularidades a fim da integração entre a indústria 4.0 e a produção, partindo desde a realização de um pedido pelo cliente até a concepção do produto, as quais incluem desde os fornecedores aos componentes de produção. Tais características correspondem a integração horizontal, engenharia de ponta a ponta e integração vertical. Sendo, a 
integração vertical uma ferramenta de melhor conectividade entre todos os elementos do ciclo de vida de um produto desde a engenharia, ao marketing. A Figura 2 corresponde a interface entre as integrações horizontal e vertical.

Figura 2

Interface Entre Integração Horizontal e Vertical
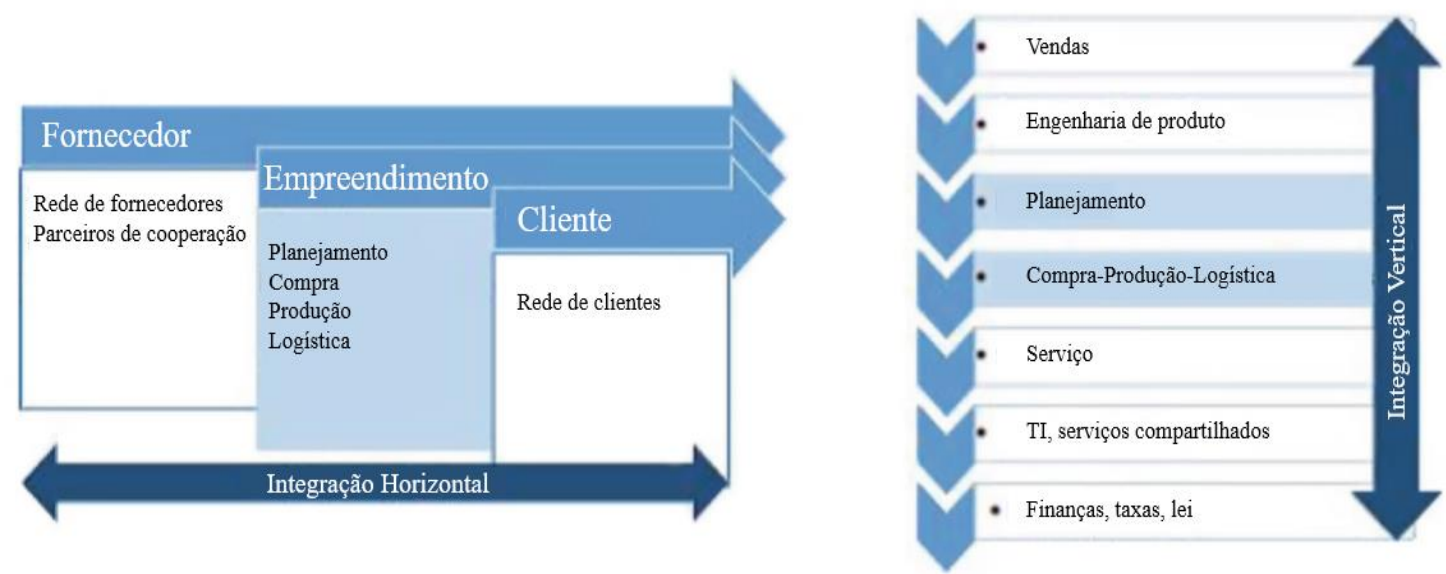

Fonte: (Wolter; Hummel; Neuber-Pohl, 2015 p. 12).

Assim, a quarta revolução industrial integra de forma crescente diversos setores produtivos, com fabricação integrada, maquinário e dispositivos conectados entre si formando um sistema (Team, 2017). Com isso, a indústria 4.0 apresenta seis atributos importantes em termos de gestão de projetos, sendo estes: A internet das coisas, sistemas cyber-físicos, Big Data, automação, inteligência artificial e computação em nuvem (Cakmakci, 2019).

Desta forma, este setor apresenta uma nova organização com objetivo de atender as necessidades individuais dos clientes (Cakmakci, 2019). Cabe ressaltar que para que um projeto apresente eficiência são necessários investimentos em tecnologias que auxiliem na realização das tarefas (Santos et al., 2018).

Segundo o PMI (2018) um projeto pode ser definido como um esforço temporário, com início, fim, escopo e recursos definidos, de modo a originar um produto ou serviço. Ainda de acordo com o PMI (2018, p. 10) o gerenciamento desse corresponde na "aplicação de conhecimentos, habilidades, ferramentas e técnicas às atividades do projeto a fim de cumprir os seus requisitos".

Para Kerzner (2018) o gerenciamento de projetos atualmente é observado como uma maneira de aumentar a competitividade de organizações elevando seus níveis de qualidade e valor agregado. 0 mesmo pode ser aplicado em situações não rotineiras em um empreendimento (Vargas, 2005) e segundo Carvalho e Rabechini Jr. (2017 p. 18) obtém eficiência "através do uso sistemático das boas 
práticas de gerenciamento, que envolvem as áreas de conhecimento, os grupos processo e o disciplinado monitoramento do ciclo de vida".

Com relação ao gerenciamento de projetos Santos et al. (2018) caracteriza-o pelo emprego de métodos, habilidades e competências, cabendo ao gestor do mesmo a toma de decisões. A gestão de projetos é realizada desde os tempos remotos, sendo capaz de reunir habilidades e recursos para alcançar um objetivo. Tais metas devem ser traçadas com precisão e incluir parâmetros como o desempenho e orçamentos (Cakmakci, 2019).

Atualmente, a internet corresponde a ferramenta mais eficaz quanto a comunicação e acesso à informação. Sistemas cibernéticos apresentam-se em números crescentes sendo possível até a criação de modelos virtuais que simulam o real. Paralelamente, o mundo dos negócios vem se transformando em um setor digital. (Kelly, 2016).

\section{Metodologia}

A metodologia utilizada nesta pesquisa compreende na utilização da análise bibliométrica e a de conteúdo. Segundo Machado (2007) uma análise bibliométrica é realizada em produções científicas buscando indicadores através de procedimentos que mensuram, mapeiam, interpretam e avaliam as produções acadêmicas. Para Amaral et al., (2019, p. 280) "a análise bibliométrica vem se mostrando muito eficaz no processo de pesquisa de temas de diversas áreas, pois possibilita sistematizar um grande volume de dados bibliométricos". Além de difundir saberes e os pensamentos dos pesquisadores (Araújo, 2006). Podendo utilizar de dados estatísticos e técnicas quantitativas para tratar os dados obtendo uma apresentação concisa dos termos analisados (Geng et al., 2017).

Por meio de análises como a coocorrência de palavras-chave, é possível observar graficamente a ocorrência simultânea das mesmas em uma amostra, seja no título, seja no resumo e palavras-chave (Eck; Waltman, 2014). Possibilitando estabelecer forças de ligação perante as expressões que coocorreram a fim de gerar um mapa do conhecimento (Franco; de Faria, 2019). Ademais, conforme Dias (2004, p.35) "palavras-chave são particularmente úteis porque podem ser interpretadas individualmente e independentemente umas das outras."

Por sua vez, com a técnica de análise de conteúdo (Júnior; Wilson, 2005) observou-se as principais temáticas abordadas na literatura. Como também, pôde-se realizar uma classificação e categorização dos diversos tipos de conteúdo com a finalidade de compará-los (Carlomagno; da Rocha, 2016) e discuti-los quanto ao gerenciamento de projetos.

Desta forma, a análise do presente trabalho dividiu-se em duas etapas. Na primeira, os dados brutos fornecidos pela base foram tratados, e com o auxílio do software Minitab 18 elaborou-se gráficos como: publicações por ano, áreas de publicação, países, entre outros. Em seguida, com o auxílio do 
software VOSviewer formulou-se uma rede de palavras-chave através da amostra oriunda da base de dados.

Ademais, o Minitab 18 mostrou-se uma ferramenta eficiente no tratamento dos dados pois permite explorar os dados com gráficos, conduzir análises estatísticas, avaliar a qualidade, planejar um experimento dentre outras coisas (Minitab, 2019).

Acrescenta se que, o VOSviewer correspondeu a uma ferramenta essencial uma vez que possibilita a construção e visualização de redes bibliométricas que podem incluir: periódicos, pesquisadores ou publicações individuais e podem ser construídas com base em citações, acoplamentos bibliográficos, cocitações ou relações de coautoria (VOSviewer, 2019).

Posteriormente, a segunda etapa, análise de conteúdo, ocorreu por meio de leitura na integra de todos os resumos encontrados na base e, porventura, leitura dos documentos completos para classificá-los segundo Cakmakci (2019) em pertencentes aos grupos: Internet das coisas, sistemas cyberfísicos, Big Data, automação, inteligência artificial e computação em nuvem. Identificando posteriormente o pilar predominante em cada trabalho.

Acrescenta-se que análise de conteúdo segundo Mozzato e Grzybovski (2011 p. 734) "é um conjunto de técnicas de análise de comunicações, que tem como objetivo ultrapassar as incertezas e enriquecer a leitura dos dados coletados". De modo a observar o que está sendo discutido sobre um tema (Vergara, 2005).

A base de dados Scopus foi utilizada nesta pesquisa, ela corresponde a um banco de dados com grande volume de resumos e citações, sendo considerada a maior do segmento, apresentando periódicos científicos, livros e anais de congressos (Elsevier, 2019). Adiciona-se que tal base permite um tratamento eficiente dos dados fornecidos permitindo que referências, citações e cocitações também sejam trabalhadas.

Em janeiro de 2020, foi realizada a busca com os termos "industry 4.0*" e "project* management", onde a base localizou por meio de títulos, resumos e palavras-chave 48 publicações, com 45 sendo escritos em inglês e três em alemão. A pesquisa englobou os documentos do tipo: Conference Paper, Article, Book Chapter, Conference Review e Short Survey e não delimitou um prazo de análise, de modo a estudar todos os documentos contidos na base até a data de pesquisa. As publicações sobre os temas estão contidas entre os anos de 2014 a 2020. Todas as publicações foram lidas, analisadas, classificadas e serão apresentadas e discutidas na seção 4. 


\section{Resultados}

\subsection{Análises Iniciais}

Diante da amostra analisada, o Gráfico 1 corresponde a quantidade de publicações durante os anos de 2014 a 2020, contando com um total de 48 documentos. A primeira publicação sobre o tema surgiu em 2014 e percebe-se que a partir de 2018 houve um grande aumento na quantidade de publicações sendo 2019 o ano com maior número contando com 16 trabalhos.

\section{Gráfico 1}

Quantidade de Publicações por Ano

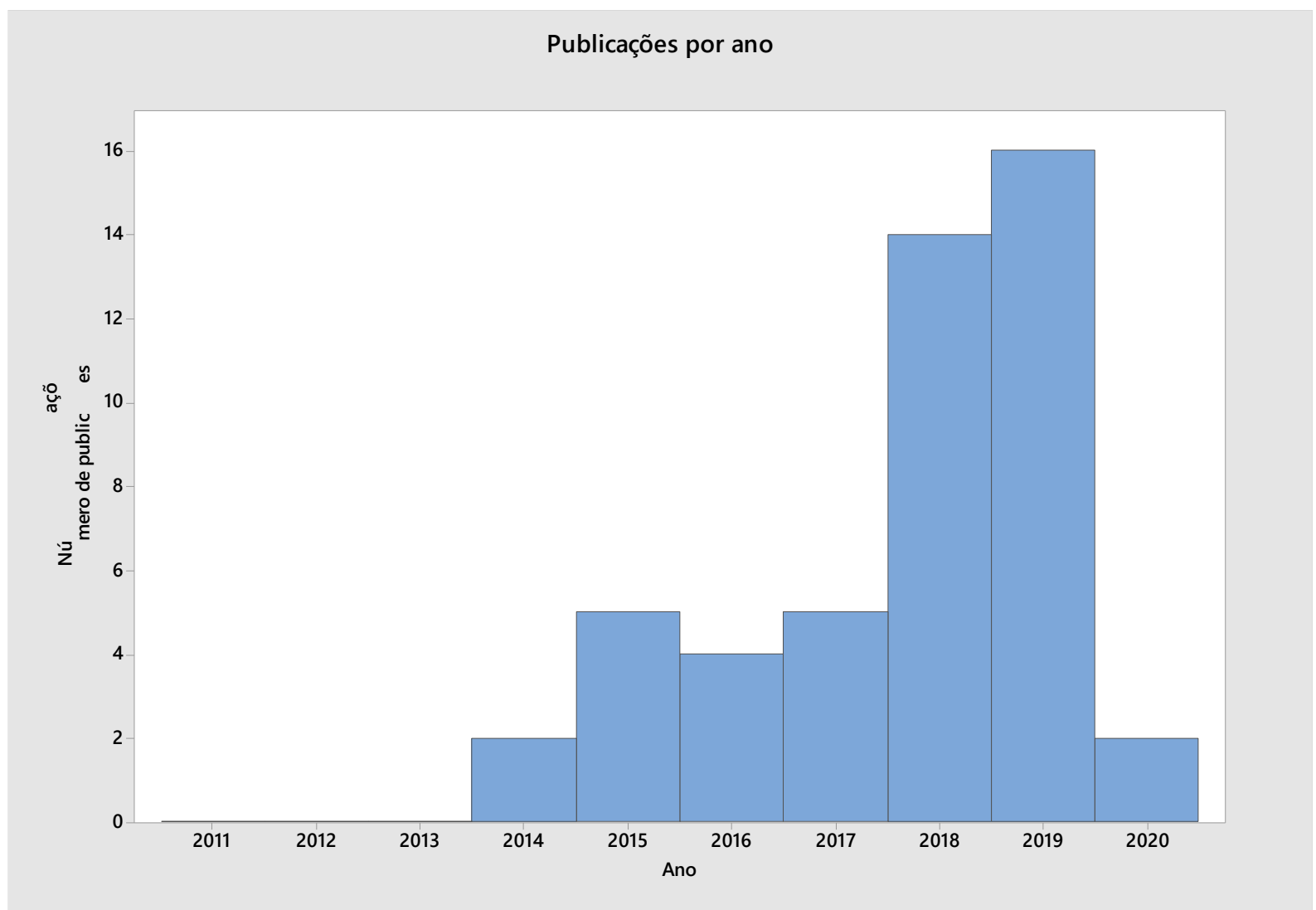

Fonte: Autores (2020).

Cabe ressaltar que a indústria 4.0 teve repercussão internacional no ano de 2011 onde foi apresentada na Alemanha, porém as pesquisas que trataram da mesma junto ao gerenciamento de projetos surgiram em 2014, tais trabalhos abordaram ferramentas inteligentes e o desenvolvimento de novos produtos. O ano de 2018 apresenta-se como um marco para o setor pois a partir dele nota-se crescente interesse no desenvolvimento de pesquisas sobre o tema.

Segundo Orth (2018) o ano de 2018 correspondeu ao ano científico onde o Ministério Federal da Educação e Pesquisa Alemão trabalhou sobre o lema "Mundos de trabalho do futuro", onde fortes 
incentivos principalmente ao desenvolvimento de pesquisas com enfoque na Indústria 4.0 aplicada ao desenvolvimento de pequenas e médias empresas foram desenvolvidas em solo alemão.

Em 2019, ano com maior quantidade de publicações (16 trabalhos), observou-se a existência de linhas de pesquisa sobretudo a respeito do desenvolvimento de novos projetos que adequem a realidade aos conceitos da indústria 4.0. Sendo também, abordados conceitos ligados a sustentabilidade na elaboração destes. Paralelamente, estudos que objetivaram analisar os graus de incertezas, efeitos de implementação, mudanças, riscos e segurança da indústria 4.0 também foram desenvolvidos neste ano.

Soma-se a isto, a grande heterogeneidade nas áreas de pesquisa da amostra, sendo Engenharia e Ciência da Computação as áreas com maior quantidade de publicações, cerca de 50\%. Ademais, áreas como: Ciências da Terra, Artes e Humanidades, e Física e Astronomia somam cerca de 3\% correspondendo as áreas de menor interesse no desenvolvimento de pesquisas que relacionam a quarta revolução industrial ao gerenciamento dos seus projetos. O Gráfico 2 apresenta as áreas de publicações da amostra analisada.

\section{Gráfico 2}

Áreas de Publicação

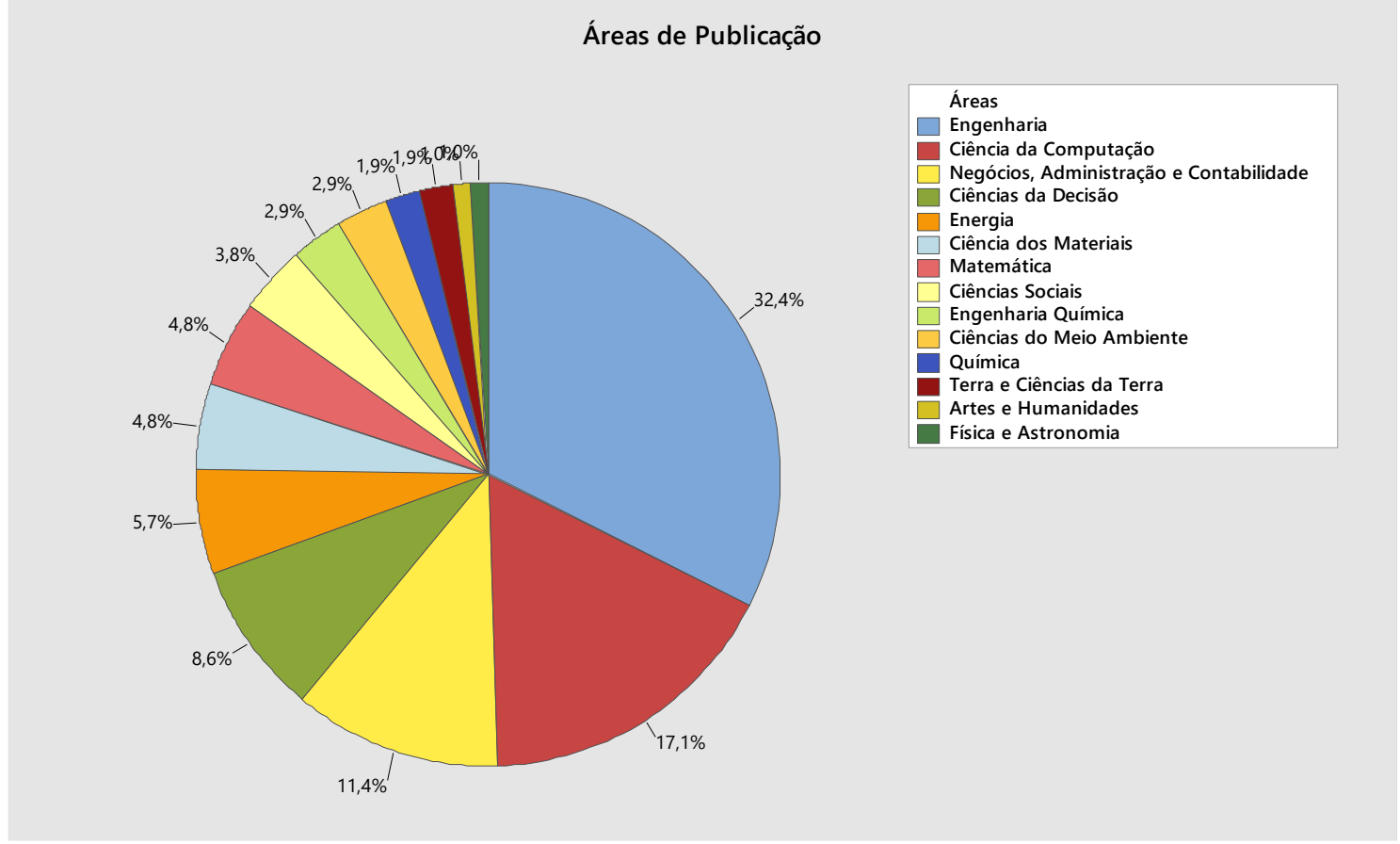

Fonte: Autores (2020).

Com relação ao cenário de países com maior número de publicações, Gráfico 3, percebe-se que a Alemanha se destaca (cerca de 10 publicações). Segundo Da Silva, Kovaleski e Pagani (2020) a Industria 4.0 já é uma realidade em tal país. Esse fato pode ser justificado pelo grande volume de investimentos 
que o setor comercial disponibiliza para o desenvolvimento de pesquisas relacionadas a Indústria 4.0, principalmente aos assuntos que envolvam segurança (Romer, 2016).

\section{Gráfico 3}

Total de Publicações por Países

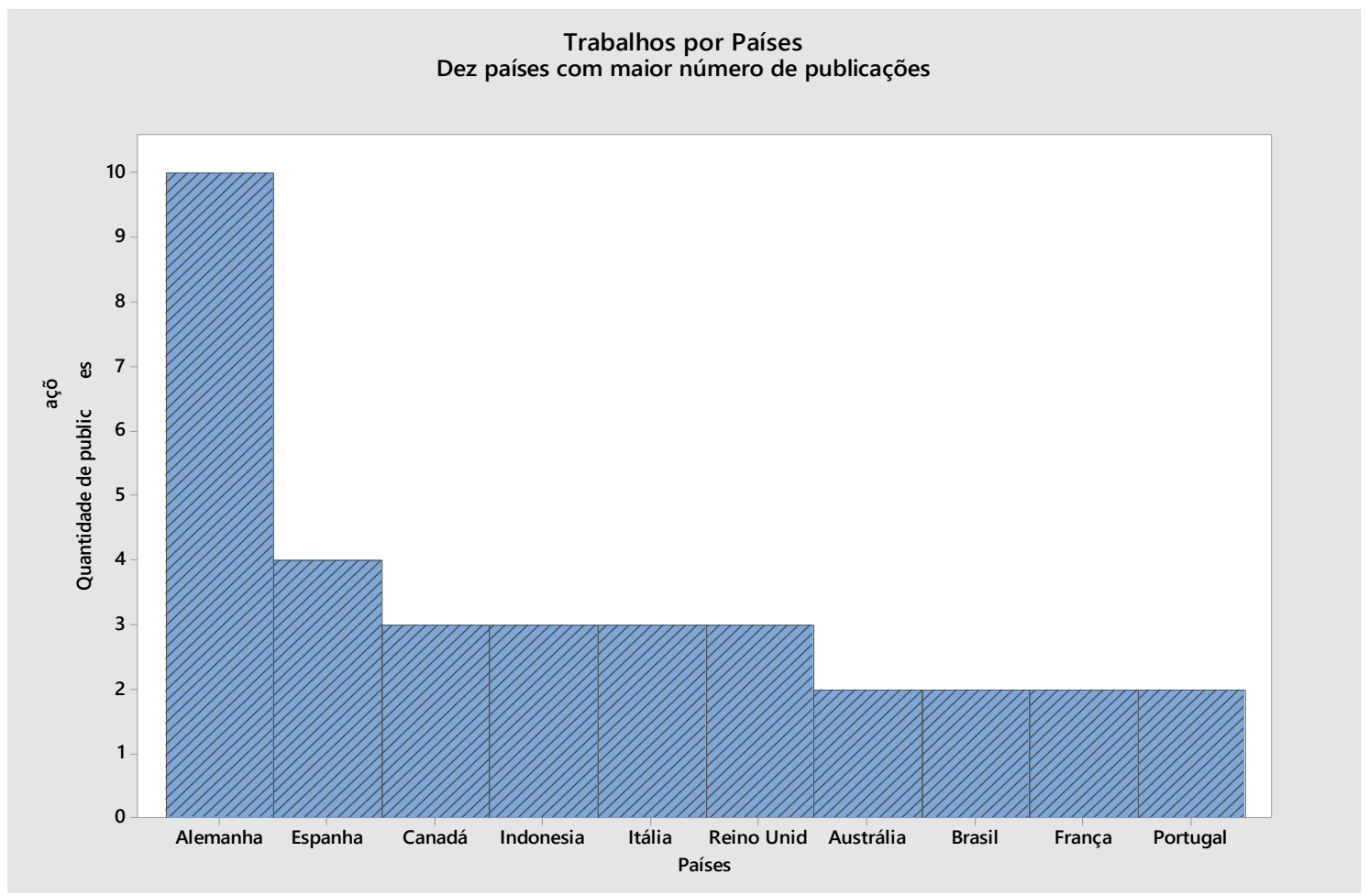

Fonte: Autores (2020).

Acrescenta-se que os desafios que cada país enfrentará quanto a implementação da Indústria 4.0 são diferentes e diretamente relacionados aos propósitos de sua manufatura na economia do futuro. Mesmo Alemanha e Itália, primeira e segunda posição em termos de tamanho da indústria, possuem diferenças como: para a Alemanha a indústria 4.0 proporciona vantagens competitivas, porém tais vantagens não são tão claras na estrutura industrial da Itália (Fiesp, 2017).

Segundo Berger (2014) é possível observar os estágios dos países levando em consideração seus índices de preparação para a Indústria 4.0 e a percentagem da indústria de transformação no PIB (medido pelo índice). Sendo que o eixo vertical leva em consideração a excelência industrial, valor agregado, abertura do setor, rede de inovação e sofisticação, e o eixo horizontal representa a medida da indústria de transformação sobre PIB. A Figura 3 corresponde a tal mensuração aplicada a União Europeia. 
Figura 3

A implementação da Indústria 4.0 na União Europeia

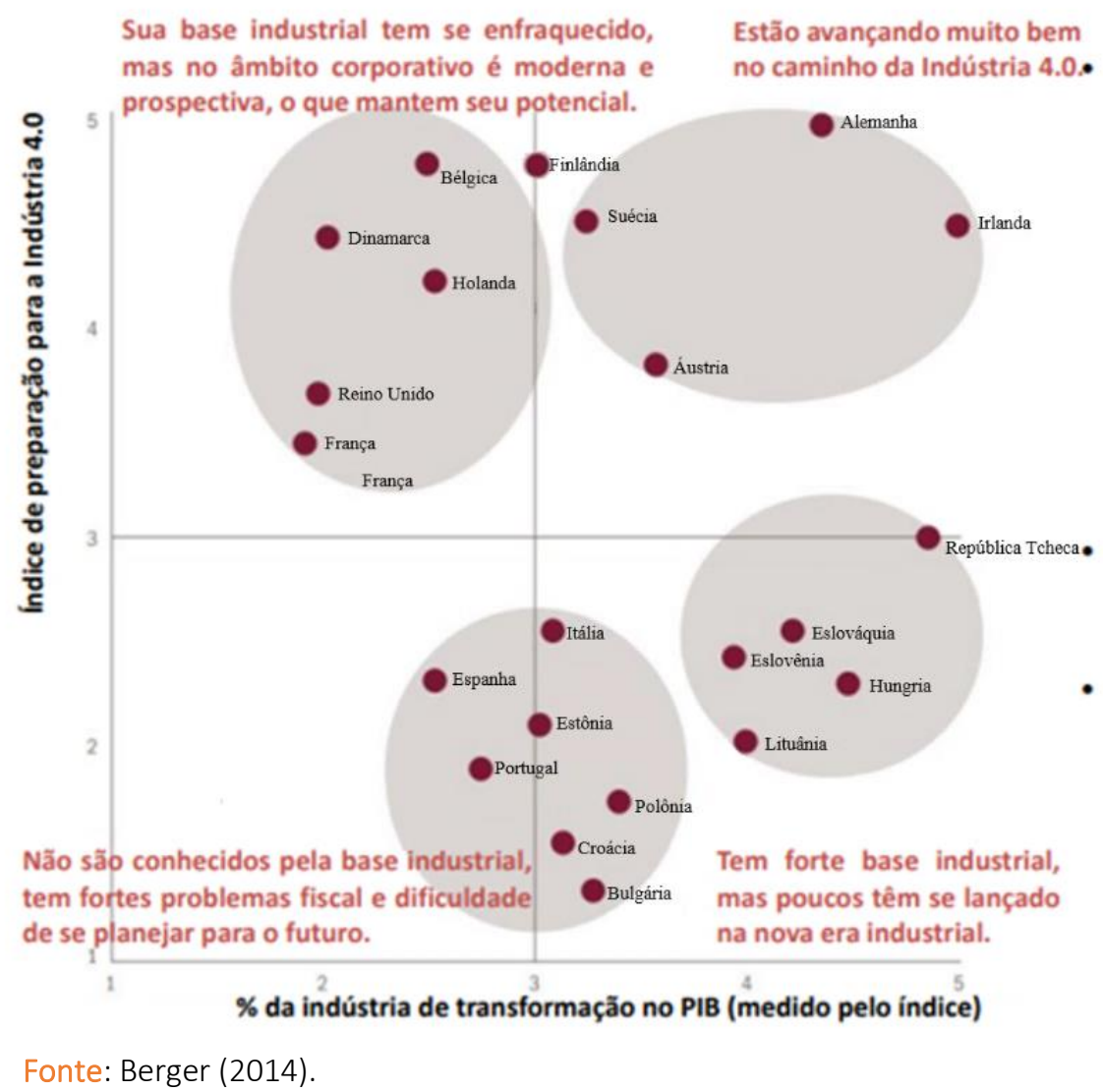

Pode-se perceber que a Alemanha representa o país mais avançado quanto a implementação do conceito 4.0, enquanto a Bulgária tem forte base industrial, porém não demonstra interesse na aplicação deste.

Quando comparadas as quantidades de publicações por país aos estudos de Berguer (2014) e Romer (2016), percebe-se que há grande empenho por parte da Alemanha em continuar sendo a líder no desenvolvimento de estudos sobre a Indústria 4.0, sobretudo aplicados ao gerenciamento de projetos. Ademais, nota-se que a Espanha, segunda colocada no ranking publicações, demonstra interesse em desenvolver os assuntos correspondentes a tais temas. O Gráfico 3 apresenta as quantidades de publicações por países contidas na amostra.

Paralelamente, nas américas, os assuntos abordados pelos pesquisadores correspondem principalmente a Engenharia e Administração onde os assuntos tratam principalmente de inovações no gerenciamento de projetos. O Canadá lidera a lista composta por três países contando com três publicações, seguido do Brasil (duas publicações) e Estados Unidos (duas publicações).

Os estudos realizados no continente asiático semelhante as américas correspondem aos mesmos campos, abordando tanto a necessidade de mudanças no conceito de gerenciamento de 
projetos e implementação de sistemas inteligentes, quanto a preparação de alunos para o futuro mercado de trabalho. Taiwan lidera com duas publicações e os demais com uma publicação por país.

Tendo em vista as publicações da base de dados SCOPUS, os trabalhos mais citados são mostrados na Tabela 1. Os documentos tratam de gerenciamento de projetos como um campo que precisa ser explorado e atualizado conforme as novas tecnologias, considerando novos dispositivos, técnicas e ferramentas.

Assim como, avaliam os cenários de gestão de suas respectivas épocas propondo medidas mitigadoras, problemas, tendências e desafios a serem implementados ora em fábricas, ora na construção de edifícios.

Analisando o número de citações, se destacam os documentos de Haddara e Elragal (2015), totalizando 23 citações, e Gentner (2016), com 13 citações. Com ambos tratando de discutir os recursos empresariais de suas épocas, com o segundo ampliando sua pesquisa para as tecnologias do futuro.

\section{Tabela 1}

Publicações Mais Citadas

\begin{tabular}{|c|c|c|c|}
\hline Autores & $\begin{array}{c}\text { Ano de } \\
\text { Publicação }\end{array}$ & Principais Abordagens & $\begin{array}{c}\text { № de } \\
\text { Citações }\end{array}$ \\
\hline $\begin{array}{l}\text { Haddara, M., } \\
\text { Elragal, A. }\end{array}$ & 2015 & $\begin{array}{l}\text { Verificar se os sistemas de planejamento de } \\
\text { recursos empresariais de } 2015 \text { estavam prontos } \\
\text { para a Fábrica do Futuro. }\end{array}$ & 23 \\
\hline Gentner, S. & 2016 & $\begin{array}{c}\text { Discutir como identificar o que já é realidade, } \\
\text { quais ideias podem se tornar realidade no } \\
\text { futuro e quais ideias permanecerão ficção } \\
\text { científica. }\end{array}$ & 13 \\
\hline $\begin{array}{l}\text { Kryvenchuk, Y., } \\
\text { Shakhovska, N., } \\
\text { Melnykova, N., } \\
\text { Holoshchuk, R. }\end{array}$ & 2019 & $\begin{array}{l}\text { Os principais indicadores de desempenho para } \\
\text { o gerenciamento de projetos. }\end{array}$ & 5 \\
\hline $\begin{array}{l}\text { Sauer, M.S., } \\
\text { Grosch, T., } \\
\text { Abele, E. }\end{array}$ & 2014 & $\begin{array}{l}\text { Apontar os problemas do gerenciamento de } \\
\text { ferramentas de hoje e descrever a abordagem } \\
\text { de projeto do uso de sistemas cyber-físicos para } \\
\text { obter maior transparência e eficiência no } \\
\text { gerenciamento de ferramentas de fabricação. }\end{array}$ & 4 \\
\hline $\begin{array}{l}\text { Yang, H.-L., Chang, } \\
\text { T.-W., Choi, Y. }\end{array}$ & 2018 & & \\
\hline
\end{tabular}




\begin{tabular}{|c|c|c|c|}
\hline Autores & $\begin{array}{c}\text { Ano de } \\
\text { Publicação }\end{array}$ & $\begin{array}{c}\text { No de } \\
\text { Citações }\end{array}$ & 3 \\
\hline $\begin{array}{c}\text { Stojanovic, V., } \\
\begin{array}{c}\text { Trapp, M., Richter, } \\
\text { R., Hagedorn, B., } \\
\text { Döllner, J. }\end{array}\end{array}$ & 2018 & $\begin{array}{r}\text { Explorar as tendências de pesquisa de fábricas } \\
\text { inteligentes em pesquisas internacionais e } \\
\text { especificamente coreanas. }\end{array}$ & $\begin{array}{c}\text { Apresentar o progresso atual da pesquisa e } \\
\text { desenvolvimento de uma plataforma orientada } \\
\text { para o serviço para a geração de } \\
\text { representações de nuvem de pontos 3D } \\
\text { semântica mente ricas de ambientes internos. }\end{array}$ \\
\hline
\end{tabular}

Fonte: Autores (2020).

\subsection{Análise de Rede}

Para a verificação da amostra a respeito de seus respectivos temas, fora realizada uma análise de a coocorrência de palavras-chave a partir do software VOSViewer.

Para a análise de palavras-chave identificou-se 499 destas, com formação de no mínimo 2 palavras, o software agrupou-as em clusters distintos separados por cor, sendo o tamanho de cada nó é proporcional a quantidade de ocorrências e suas distâncias ao grau de relação entre itens. Contudo, os termos mais utilizados foram: Project Management, Industry 4.0 e information management.

Figura 4

\section{Rede Coocorrência de Palavras-Chave}

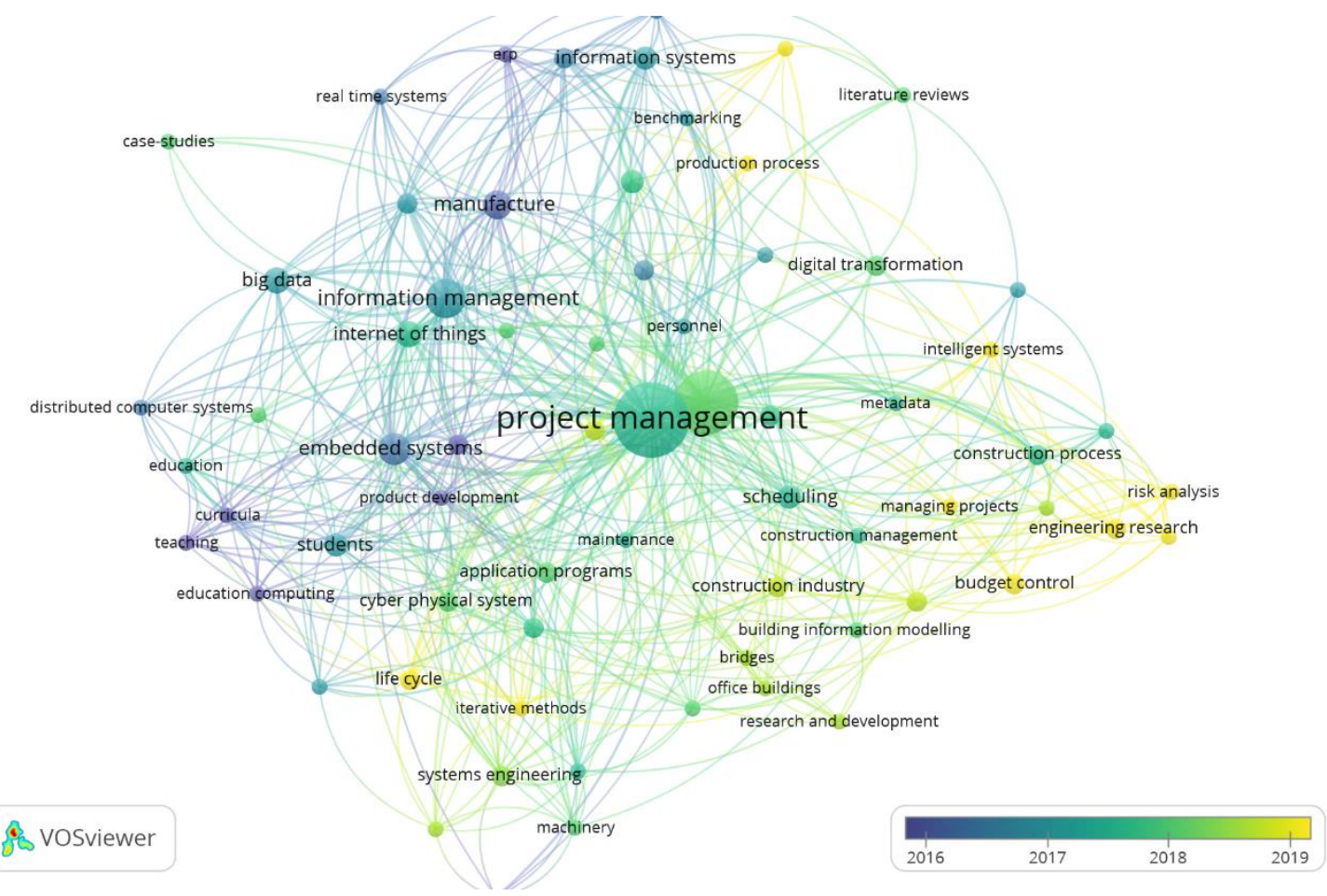

Fonte: Autores (2020). 
Por meio da escala de cores é possível observar a existência de certas tendências de pesquisa para o futuro, sobretudo que relacionam gestão de projetos a indústria 4.0, sendo estas correspondentes a: análises de risco, controle orçamental, investigações de engenharia e avaliação de riscos. Os clusters de maior interesse desta pesquisa correspondem aos de número 2 e 3, Indústria 4.0 e gestão de projetos. A Figura 5 apresenta os clusters 2 e 3.

Figura 5

Clusters 2 e 3

\section{Cluster 2}

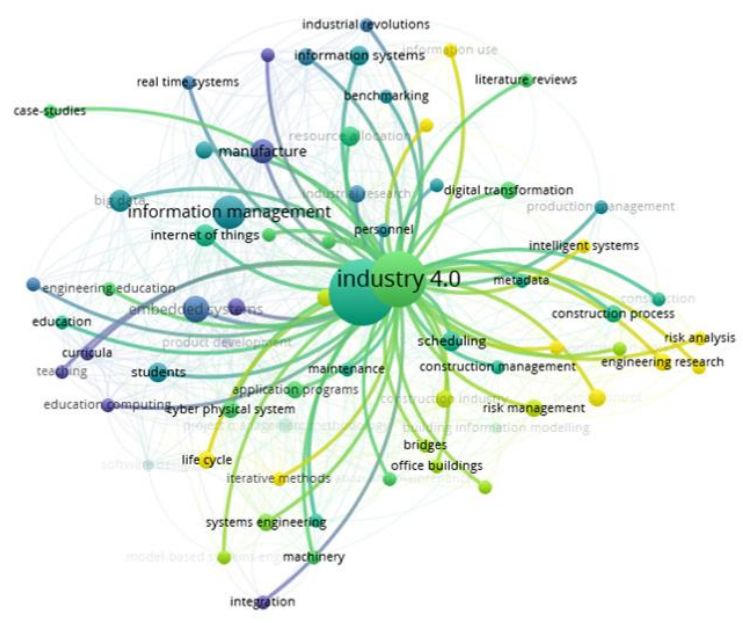

\section{Cluster 3}

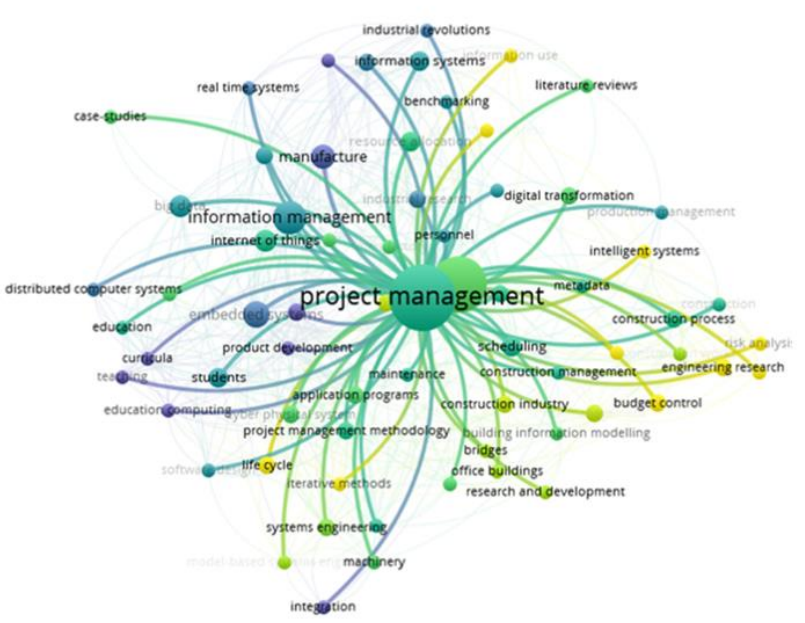

Fonte: Autores (2020).

O cluster 2 apresenta correlações distribuídas ao longo de todos os anos sendo associado a todos os demais, revelando que os pesquisadores desenvolvem trabalhos desde o âmbito educacional, a inteligências artificiais. As principais ligações deste são relacionadas gestão de informações, sistemas cyber-físicos e sistemas de informação, ou seja, o conceito 4.0 prioriza o desenvolvimento de tais áreas.

Por sua vez, o cluster 3 similar ao anterior tanto na distribuição ao longo do tempo, quanto nas associações com os demais, revela que a gestão de projetos prioriza o gerenciamento de informações, manufatura e sistemas incorporados. O gerenciamento de informações está fortemente atrelado ao termo big data inferindo o grande número de dados envolvidos na gestão de projetos. Por sua vez, a manufatura associa-se principalmente a aos sistemas incorporados sugerindo a crescente modernização das fábricas. Por fim, sistemas incorporados apresentam ligação direta ao termo indústria 4.0 expondo que a quarta revolução industrial representa uma base para o desenvolvimento de dispositivos integrados que proporcionem um melhor gerenciamento de projetos. 
Por fim, os demais clusters dissertam sobre deferentes temas, o primeiro, exprime que os sistemas microprocessados, estudados principalmente em 2016, são utilizados como ferramentas educacionais sobre computação que preparam estudantes para as mudanças futuras do mercado de trabalho. O quarto apresenta as tendências da manufatura como sistemas em tempo real, alocação de recursos e internet das coisas. O posterior trabalha a gestão de informações discorrendo sobre as transformações digitais, os grandes volumes de dados e os sistemas de informação. Por sua vez, o sexto refere que sistemas incorporados apresentam grande volume de informações, das quais segundo as coocorrências contém altos riscos se não forem administrados corretamente. Por fim, o sétimo, evidencia os métodos interativos e a corrida para o desenvolvimento de novas tecnologias. Os clusters 1 e 4; 5 e 6; e 7 estão contidos nas Figuras 6, 7 e 8, respectivamente.

Figura 6

Clusters 1 e 4

Cluster 1

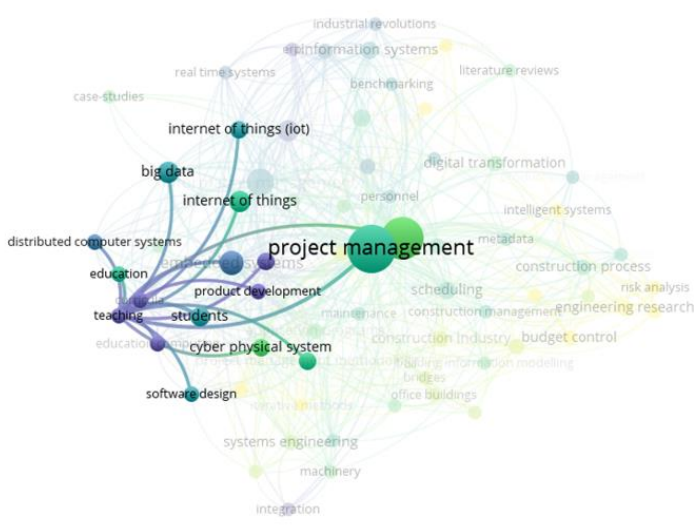

Fonte: Autores (2020).

\section{Cluster 4}

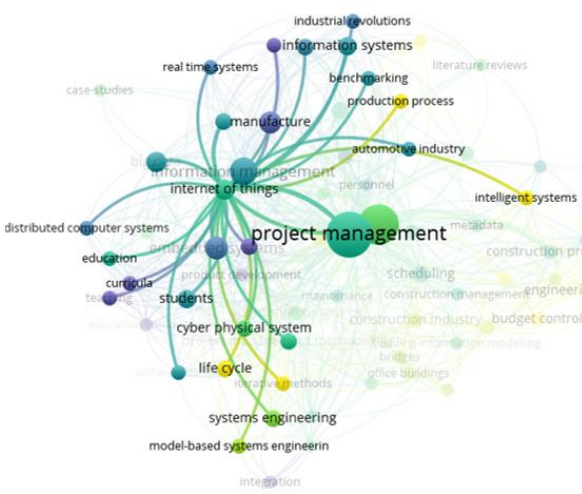


Figura 7

\section{Clusters 5 e 6}
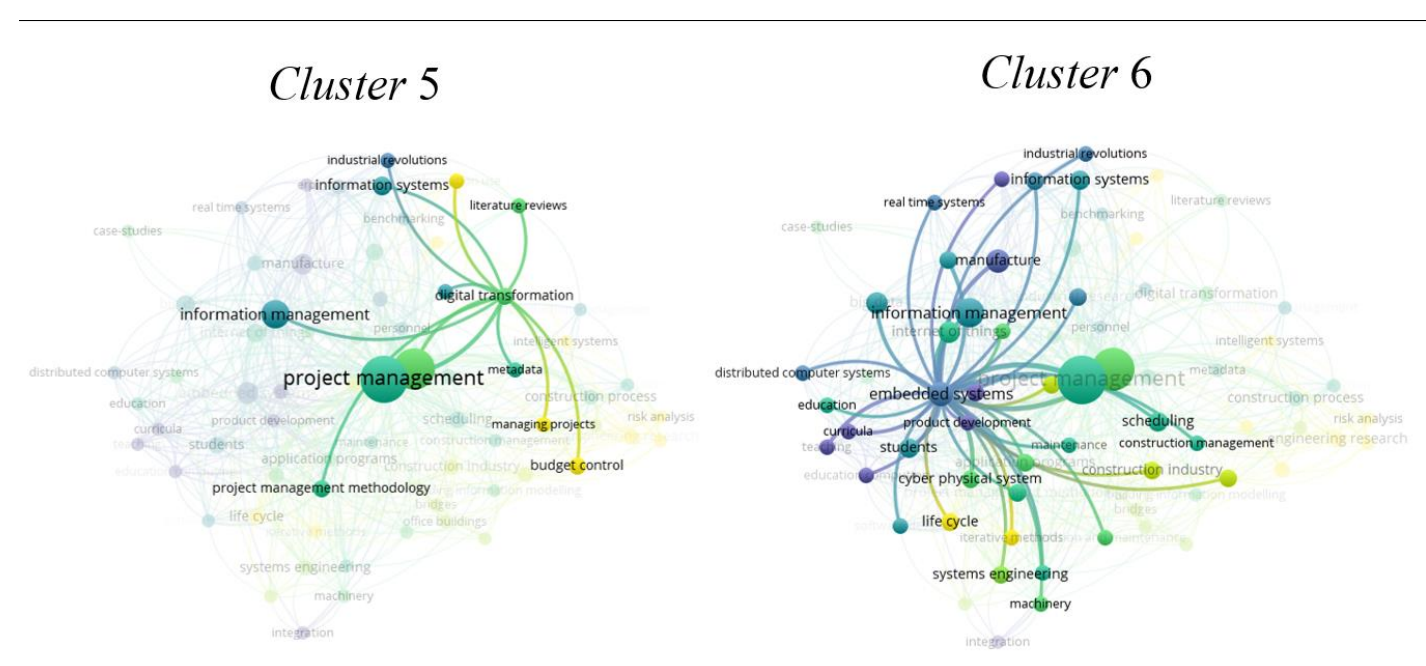

Fonte: Autores (2020).

Figura 8

\section{Cluster 7}

\section{Cluster 7}

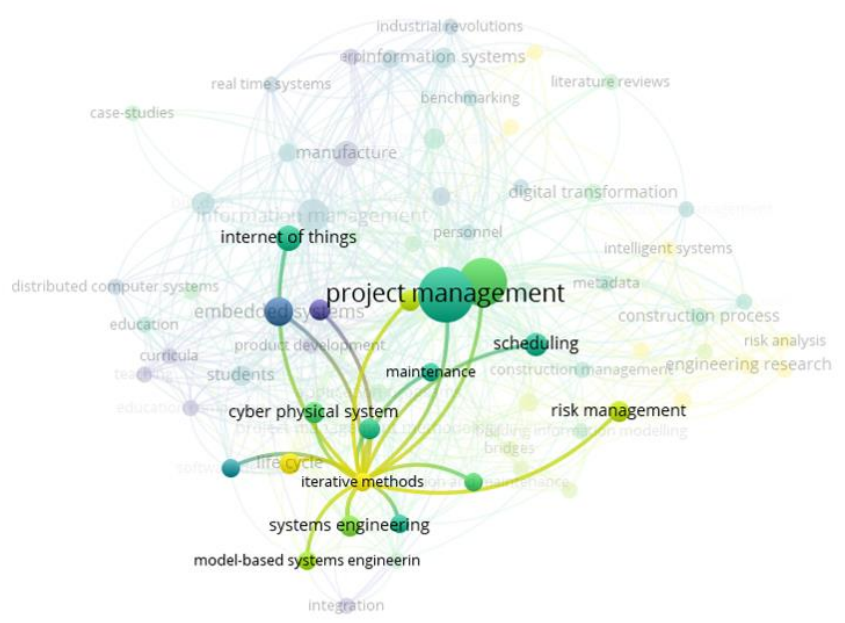

Fonte: Autores (2020).

\subsection{Os principais pilares da indústria 4.0 para o gerenciamento de projetos}

Segundo Cakmakci (2019) o conceito 4.0 considera seis pilares principais: Internet das coisas, sistemas cyber-físicos, Big Data, automação, inteligência artificial e computação em nuvem. Sendo tratados no âmbito do gerenciamento de projetos, os documentos foram apontados em uma das seis 
perspectivas da quarta revolução industrial, conforme apresentado na Tabela 3. Sendo assim, as categorias podem ser descritas como:

- Internet das coisas - Documentos que tratam o gerenciamento de projetos com o uso de objetos físicos utilizados na produção conectados via software incorporado, sensores, dispositivos eletrônicos e de rede e Internet (ILX, 2017).

- Sistemas Cyber-Físicos - Pesquisas que desenvolveram sistemas computacionais de gestão de projetos que ligam o mundo físico aos seus processos em andamento, e que ofereceram e cooperaram com serviços de processamento e acesso de dados. (Monostori et al., 2016)

- Big Data - Pesquisas que abordaram o gerenciamento de projetos com grandes quantidades de dados oriundos da implementação de máquinas, robôs, logística e feedback do usuário, entre outros. (Leei; Bagheri; Kao, 2015)

- Automação - Estudos que analisaram a gestão de projetos junto ao uso de tecnologias como: impressão 3D, tecnologia de nuvem, processos de produção são acelerados, a qualidade é melhorada e desempenho é aumentada (Kagermann; Lukas; Wahlster, 2011)

- Inteligência Artificial - Registros que discutiram a utilização de algoritmo de aprendizado de máquinas para simular a inteligência humana, aprender automaticamente e melhorar suas condições de operação, junto ao gerenciamento de projetos (Isaacs et al., 2017)

- Computação em Nuvem - Trabalhos que dissertaram sobre o gerenciamento de projetos a partir da utilização de dados em tempo real obtidos por sensores e conversores distribuídos por todo o ciclo de produção resultando em decisões precisas tomadas de forma contínua e autônoma (Chen, 2017)

\subsubsection{Análises}

Conforme ilustra o Quadro 1, 40 trabalhos puderam ser enquadrados nos pilares propostos por Cakmakci (2019), sendo que 55\% destes relacionados ao gerenciamento de projetos junto ao conceito 4.0 centraram-se no desenvolvimento de sistemas cyber-físicos, sendo esses, resultado da crescente implantação de tecnologias nos projetos, como sensores de alta capacidade que aliados a instrumentos computacionais que estão revolucionando a comunicação (Rajkumar et al., 2010). A utilização deste grande volume de informações contribui para a realização de uma melhor gestão em todo o ciclo de vida de um projeto, contribuindo tanto para o controle, quanto o monitoramento desses.

Soma-se a isso, o fato de os documentos tratarem desde a criação de sistemas para fábricas, a formulação de metodologias de ensino inovadoras, com o setor privado sendo o principal alvo dos pesquisadores. Vale salientar, que ambas as pesquisas desenvolvidas em 2020 estão enquadradas nessa categoria, o que revela tendências para futuras pesquisas sobre o tema.

Seguido por Big Data com 20\% dos trabalhos abordaram a gestão de projetos dentro da quarta revolução industrial como um gerenciamento de grande número de informações provindas de sensores, aparelhos e máquinas. Tal gestão segundo (Wang, 2015) representa não apenas uma forma de melhoramento dos efeitos, como também, melhoramento do ambiente tornando-o mais confiável. 
Acrescenta-se que este fenômeno sinaliza que tal área da administração caminha para uma evolução sem precedentes de modo a anteceder eventos, comportamentos e tendências. Segundo Chen, Mao e Liu (2014) tal volume de dados é tamanho que não seria possível perceber, adquirir, gerenciar ou processá-lo através de ferramentas tradicionais de TI.

Em conjunto, automação (10\%) abordaram-se principalmente questões sobre o gerenciamento e automatização de tarefas dentro dos processos de desenvolvimento de máquinas. De forma a melhorar a competitividade dos projetos em âmbito global. Com a utilização de máquinas o gestor pode ter maior controle sobre toda a linha de produção, além de avaliar e prever com maior precisão os possíveis cenários futuros.

As pesquisas sobre Internet das Coisas (7,5\% das pesquisas) trataram a gestão de projetos através de máquinas inteligentes que segundo (Alsaadoun, 2019) devido a sua rápida evolução corresponde a uma ferramenta fundamental para a evolução provável da Industria 4.0.

Ao discutir-se tais pesquisas em uma ótica de gestão de projetos, a percebe-se certa tendência a substituição das máquinas contemporâneas operadas por humanos por autossuficientes, a exemplo da construção civil, onde os processos caminham para uma execução autônoma, sem presença direta de um operador. Entretanto, fatores como integridade da produção, disponibilidade e confidencialidade apresentam-se como desafios a serem enfrentados pelos gestores de projetos. Segundo Pacchini et al. (2020) segurança corresponde a maior barreira quanto a implementação da Industria 4.0 na indústria.

Computação em nuvem (5\%) enfatizaram principalmente a utilização de dados em tempo real, como também, o processamento de dados na nuvem. Quanto mais rápido uma informação que trate de falha, problema ou conflito chegar até o gestor, melhor e menos danosa ao projeto tenderá a solução escolhida pelo mesmo, a exemplo tem-se o trabalho de Braga e Raupp (2020) que discutiu o alinhamento entre sua gestão de custos e tomada de decisões no qual um sistema de gestão online apresentou-se de grande valia na condução de uma organização.

Por fim, o documento que abordou inteligência artificial criou um sistema de gestão de qualidade inteligente que aprende e se adapta à mudança do sistema de fabricação flexível, revelando assim a criação de um possível "gestor virtual". 


\section{Quadro 1}

Classificação dos Trabalhos Segundo os Pilares Analisados

\begin{tabular}{|c|c|c|}
\hline Pilar & № Referências & Referências \\
\hline Internet das Coisas & 3 & $\begin{array}{l}\text { (Alsaadoun, 2019), (Dallasega; Frosolini; Matt, 2016), (De } \\
\text { Oliveira Cordeiro; Deschamps; De Lima, 2017) }\end{array}$ \\
\hline Sistemas Cyber-Físicos & 22 & $\begin{array}{l}\text { (Amran; Saraswati; Harahap, 2019), (Chern et al., 2017), } \\
\text { (Rauch et al., 2018), (Padillo et al., 2018), (Funk et al., } \\
\text { 2015), (Jovanović et al., 2015), (M Cakmakci, 2019), } \\
\text { (Aoyama et al., 2016), (Emmer et al., 2017), (C. H. Yang, } \\
\text { 2018), (Haddara; Elragal, 2015), (Yin; Qin, 2019), (Chofreh } \\
\text { et al., 2020), (Villar-Fidalgo et al., 2018), (Hendradewa, } \\
\text { 2019), (Sauer; Grosch; Abele, 2014), (Miranda; Sugarindra, } \\
\text { 2019), (Pereira; Ferreira; Silva, 2019), (Trusculescu; } \\
\text { Draghici; Albulescu, 2015), (Hasibovic; Tanovic, 2019), } \\
\text { (Albrecht; Anderl, 2014), (Salehi, 2020) }\end{array}$ \\
\hline Big Data & 8 & $\begin{array}{l}\text { (Wang, 2015), (Hui, 2018), (Moeuf et al., 2019), (Goger; } \\
\text { Bisenberger, 2018), (Cividino et al., 2019), (Tountopoulos; } \\
\text { Kavakli; Sakellariou, 2018), (Benali et al., 2018), (Low; Gao; } \\
\text { Ng, 2019) }\end{array}$ \\
\hline Automação & 4 & $\begin{array}{l}\text { (Rivera et al., 2017), (Ahmad et al., 2018), (Stojanovic et } \\
\text { al., 2018), (Kryvenchuk et al., 2019) }\end{array}$ \\
\hline Inteligência Artificial & 1 & (Bihi; Luwes; Kusakana, 2018), \\
\hline Computação em Nuvem & 2 & (Binner, 2016), (Schmitt, 2019) \\
\hline
\end{tabular}

Fonte: Autores (2020).

Entretanto, 8 dos trabalhos contidos na amostra não puderam ser classificados dentro dos 6 pilares, pois tratavam de assuntos específicos da gestão de projetos na Indústria 4.0, o Quadro 2 apresenta os trabalhos e um breve resumo das atividades desenvolvidas pelos respectivos autores destes.

\section{Quadro 2}

Trabalhos que não Se Enquadraram nos Pilares Analisados

\begin{tabular}{|l|l|}
\hline \multicolumn{1}{|c|}{ Autores } & \multicolumn{1}{c|}{ Foco } \\
\hline (Zaouga; Rabai; Alalyani, 2019) & $\begin{array}{l}\text { Tratou do aprimoramento de uma representação } \\
\text { compartilhada na gestão de recursos humanos. }\end{array}$ \\
\hline (Zgodavova; Sutoova; Cicka, 2019) & Apresentou novos projetos para a indústria 4.0. \\
\hline (Piengang; Beauregard; Kenné, 2017) & $\begin{array}{l}\text { Revisitou estudos anteriores e otimizou a tomada } \\
\text { de decisões nas fabricas. }\end{array}$ \\
\hline (Barata; da Cunha; Coyle, 2018) & $\begin{array}{l}\text { Abordou a implementação de projetos-piloto na } \\
\text { transformação digital. }\end{array}$ \\
\hline (H.-L. Yang; Chang; Choi, 2018) & Verificou as tendências de fabricas inteligentes. \\
\hline
\end{tabular}




\begin{tabular}{|l|l|}
\hline $\begin{array}{l}\text { ("7th International Conference on Software } \\
\text { Process Improvement, CIMPS 2018," 2019) }\end{array}$ & $\begin{array}{l}\text { Processo com } 26 \text { documentos distribuídos sobre } \\
\text { os diversos temas analisados. }\end{array}$ \\
\hline (Beller et al., 2018) & $\begin{array}{l}\text { Abordou projetos pequenos para facilitar a } \\
\text { avaliação da prova de projetos conceituais e sua } \\
\text { transição para os de maior escala. }\end{array}$ \\
\hline (Gentner, 2016) & $\begin{array}{l}\text { Discutiu e verificou quais ideias poderiam se tornar } \\
\text { realidade e quais permanecerão ficção cientifica. }\end{array}$ \\
\hline
\end{tabular}

Fonte: Autores (2020).

\section{Conclusões}

O presente trabalho teve como objetivo a realização de uma investigação nas publicações que concatenaram gerenciamento de projetos e Indústria 4.0 no período de 2014 à 2020, a fim de analisar, por meio de um estudo bibliométrico e revisões da literatura, como as produções internacionais relacionam a gestão de projetos com a utilização de tecnologias provindas da quarta revolução industrial.

Pode-se constatar oscilações no número de documentos que trataram a gestão de projetos com o uso de novas tecnologias, com média de 8 documentos publicados ao ano, sendo crescente desde 2017 o número de publicações, e 2019 correspondendo ao ano com maior número de documentos produzidos.

A Alemanha obteve principal destaque nos campos abordados no presente artigo, sendo este o país com maior soma de artigos publicados, cerca de 10 documentos. O número de publicações pode ser explicado devido ao fato de tal país a fonte do conceito 4.0, abordado internacionalmente em 2011, como também, pelos massivos investimentos no desenvolvimento de pesquisas sobre o tema pelo setor privado alemão.

A concorrência acirrada entre as empresas do setor privado e os benefícios da implantação dos instrumentos advindos da Quarta Revolução Industrial promovem o desenvolvimento de pesquisas, sobretudo em relação ao gerenciamento de projetos em vários países e resultam na criação e ocupação de fábricas inteligentes.

Paralelamente, percebe-se que os desafios para a implementação da Indústria 4.0 variam de acordo com cada país, devendo-se levar em consideração o estágio de desenvolvimento das bases industriais, origem dos principais investidores e a existência de políticas públicas que incentivem o desenvolvimento de pesquisas sobre o tema.

A área de Engenharias apresentou a maior quantidade de publicações sugere que os aspectos gerenciais de empresas privadas são os principais fomentadores de pesquisas sobre gestão de projetos. A segunda área que mais abordou tal tema foi Ciência da Computação, os estudos desta enfocaram principalmente o desenvolvimento de sistemas cyber-físicos para o controle de dados e melhoramento 
da produção. Negócios, Administração e Contabilidade como terceira, pode significar que o próprio setor administrativo investe em pesquisas de melhoria para ele.

Ademais, pode-se perceber que o setor privado promove o desenvolvimento de diferentes pesquisas de gerenciamento, como também, melhoramento dos sistemas já existentes com a implementação de computação em nuvem e internet das coisas. Dessa forma a utilização de tecnologias corresponde a chave para o melhoramento dos conceitos de administração de projetos, atendendo as gradativas expectativas populacionais.

Esta pesquisa contribui para a teoria quando identifica os principais trabalhos sobre o tema, e destaca que nos anos de 2018 e 2019, notou-se um crescimento na quantidade de publicações sobre o tema revelando oportunidade para futuras pesquisas. Ademais, pode-se perceber que o setor privado promove o desenvolvimento de diferentes pesquisas de gerenciamento, como também, melhoramento dos sistemas já existentes com a implementação de computação em nuvem e internet das coisas.

Para a prática organizacional, contribui com a percepção dos desafios para a implementação da Indústria 4.0, variantes de acordo com cada país, devendo-se levar em consideração: o estágio de desenvolvimento das bases industriais, origem dos principais investidores e a existência de políticas públicas que incentivem o desenvolvimento de pesquisas sobre o tema.

Por fim, os resultados deste trabalho apresentam fator limitante relacionado a quantidade de publicações examinadas, sendo assim, os resultados não podem ser ampliados e universalizados. Novos estudos podem ser desenvolvidos explorando outras bases, amostras maiores e englobar tanto o setor privado, quanto o público.

\section{Referências}

7th International Conference on Software Process Improvement, CIMPS 2018. (2019). Advances in Intelligent Systems and Computing; Vol. 865. Recuperado em:

https://www.scopus.com/inward/record.uri?eid=2-s2.0-

85054840742 andpartnerlD=40andmd5=41456418eecbaed2ec251cf2abec17df. Acesso: 05 jan. 2020.

Ahmad, R.; Masse, C.; Jituri, S.; Doucette, J.; Mertiny, P. (2018). Alberta Learning Factory for training reconfigurable assembly process value stream mapping. Procedia Manufacturing; 23; 237242, https://doi.org/10.1016/j.promfg.2018.04.023.

Albrecht, K.; Anderl, R. (2014). Applying actual development progress into education. ASME 2014 12th Biennial Conference on Engineering Systems Design and Analysis; ESDA 2014; 1, Doi: https://doi.org/10.1115/ESDA2014-20472.

Alsaadoun, O. (2019). A cybersecurity prospective on industry 4.0: Enabler role of identity and access management. International Petroleum Technology Conference 2019; IPTC 2019. Recuperado em: https://www.scopus.com/inward/record.uri?eid=2-s2.085064114317andpartnerID=40andmd5=a1b671b894610170097df3586b41f6ef. Acesso em: 
06 jan. 2020.

Amaral, É. P.; Mergulhão, R. C.; Akim, É. K.; Figueiredo, R. A.; Farrapo Junior, A. C. (2019). Uso da análise bibliométrica nos anais do ENEGEP de 2008 a 2016. Exacta; 17(4); 273-282, Doi: https://doi.org/10.5585/exactaep.v17n4.8244

Amran, T. G.; Saraswati, D.; Harahap, E. F. (2019). Evaluating Storage Tank Cap 10000L Manufacturer by Using Lean Project Management. IOP Conference Series: Materials Science and Engineering; 528(1), Doi: https://doi.org/10.1088/1757-899X/528/1/012052

Anderl, R. (2015). Industrie 4.0-technological approaches; use cases; and implementation. AtAutomatisierungstechnik; 63(10); 753-765.

Aoyama, K.; Mizushima, T.; Hiro, Y.; Oizumi, K. (2016). Advanced management of shipbuilding construction using monitoring system and job shop simulation. PRADS 2016 - Proceedings of the 13th International Symposium on PRActical Design of Ships and Other Floating Structures. Recuperado em: www.scopus.com/inward/record.uri?eid=2-s2.085026483379andpartnerID=40andmd5=0b65e6748421ed5ddb6774207c08bf75. Acesso em: 05 jan. 2020.

Araújo, C. A. A. (2006). Bibliometria: evolução histórica e questões atuais. Em Questão; 12(1); 11-32.

Barata, J.; da Cunha, P. R.; Coyle; S. (2018). Guidelines for using pilot projects in the fourth industrial revolution. ACIS 2018 - 29th Australasian Conference on Information Systems. Recuperado em: https://www.scopus.com/inward/record.uri?eid=2-s2.0-

85066341171andpartnerID=40andmd5=fcddf6b35e94b895e1f5b3800a4558e8. Acesso em: 05 jan. 2020.

Beller, C. S.; Muncinelli, G. De Lima; E. P.; Deschamps, F. (2018). Assessing industry 4.0 technology deployment projects. IISE Annual Conference and Expo 2018; 803-807. Recuperado em: https://www.scopus.com/inward/record.uri?eid=2-s2.085054007472andpartnerlD=40andmd5=fb69c951ee949c5339a3db9d1a220af7. Acesso em: 05 jan. 2020.

Benali, I.; Dafflon, B.; Bentaha, L.; Moalla, N. (2018). Emergent technologies for inter-enterprises collaboration and business evaluation. International Conference on Software; Knowledge Information; Industrial Management and Applications; SKIMA; 2017-Decem, Doi: https://doi.org/10.1109/SKIMA.2017.8294094

Berger, R. (2014). INDUSTRY 4.0-The new industrial revolution/ Alle Publikationen/ Medien/ Roland Berger.

Bihi, T.; Luwes, N.; Kusakana, K. (2018). Innovative Quality Management System for Flexible Manufacturing Systems. 2018 Open Innovations Conference; OI 2018; 40-46, Doi: https://doi.org/10.1109/OI.2018.8535610

Binner, H. F. (2016). Paradigm shift in organizational development: Conditions for a process-oriented value culture. ZWF Zeitschrift Fuer Wirtschaftlichen Fabrikbetrieb; 111(1-2); 19-22, Doi: https://doi.org/10.3139/104.111457

Blanco, R.; Oliveira, J. C. De. (2018). COMPETÊNCIAS DE GESTORES DE PROJETOS PARA A INDÚSTRIA. 
Braga, L. N. P.; Raupp, F. M. (2020). Gestão de Custos Alinhada à Tomada de Decisão: o Caso de uma Empresa Incubada de Base Tecnológica. Revista de Negócios; 25(3); 22-44.

Cakmakci, M. (2019). Interaction in project management approach within industry 4.0. Lecture Notes in Mechanical Engineering; pp. 176-189, Doi: https://doi.org/10.1007/978-3-030-18715-6_15

Cakmakci, Mehmet. (2019). Interaction in Project Management Approach (Vol. 1), Doi: https://doi.org/10.1007/978-3-030-18715-6

Carlomagno, M. C.; da Rocha, L. C. (2016). Como criar e classificar categorias para fazer análise de conteúdo: uma questão metodológica. Revista Eletrônica de Ciência Política; 7(1).

Carvalho, M. M. de; Rabechini Jr., R. (2017). Fundamentos em gestão de projetos: construindo competências para gerenciar projetos (4a ed.). São Paulo: Atlas.

Cavalcanti, V. Y. S. De L.; Souza, G. H. De; Sodré, M. A. C.; Abreu, M. S. D. De; Maciel, T. Da S.; Silva, J. M. De A. (2018). Indústria 4.0: Desafios E Perspectivas Na Construção Civil. Revista Campo do Saber; 4; 146-158.

Chen, M.; Mao, S.; Liu, Y. (2014). Big data: A survey. Mobile Networks and Applications; 19(2); 171209.

Chen, Y. (2017). Integrated and intelligent manufacturing: Perspectives and enablers. Engineering; 3(5); 588-595.

Chern, M. J.; Hsia, H. J.; Chen, S. F.; Chen, H. L.; Kuan, W. S. (2017). Education of innovation and creativity thinking on Industry 4.0 course and project. Proceedings of the 45th SEFI Annual Conference 2017 - Education Excellence for Sustainability; SEFI 2017; 1362-1369. Recuperado em: https://www.scopus.com/inward/record.uri?eid=2-s2.0-

85034747747 andpartner ID=40andmd5=a9e295dc3c42ea5302e848be1df1da89. Acesso em: 06 jan. 2020.

Chofreh, A. G.; Goni, F. A.; Klemeš, J. J.; Malik, M. N.; Khan, H. H. (2020). Development of guidelines for the implementation of sustainable enterprise resource planning systems. Journal of Cleaner Production; 244, Doi: https://doi.org/10.1016/j.jclepro.2019.118655

Cividino, S.; Egidi, G.; Zambon, I.; Colantoni, A. (2019). Evaluating the degree of uncertainty of research activities in Industry 4.0. Future Internet; 11(9), Doi: https://doi.org/10.3390/fi11090196

Da Silva, V. L.; Kovaleski, J. L.; Pagani, R. N. (2020). Influências do conceito e das tecnologias da indústria 4.0 no ambiente industrial. Exacta; 18(2); 420-437, Doi: https://doi.org/10.5585/exactaep.v18n2.10487

Dallasega, P.; Frosolini, M.; Matt, D. T. (2016). An approach supporting real-time project management in plant building and the construction industry. Proceedings of the Summer School Francesco Turco; 13-15-Sept; 247-251. Recuperado em: https://www.scopus.com/inward/record.uri?eid=2-s2.085006001520andpartnerID=40andmd5=90d57d53903bd9c5e6b9afd53e747953. Acesso em: 06 jan. 2020. 
Davies, R. (2015). Digitalisation for productivity and growth. (September). Recuperado em: http://www.europarl.europa.eu/RegData/etudes/BRIE/2015/568337/EPRS_BRI(2015)568337_ EN.pdf

Davis, J.; Edgar, T.; Porter, J.; Bernaden, J.; Sarli, M. (2012). Smart manufacturing ; manufacturing intelligence and demand-dynamic performance. Computers and Chemical Engineering; 47; 145-156, Doi: https://doi.org/10.1016/j.compchemeng.2012.06.037.

De Oliveira Cordeiro, G.; Deschamps, F.; De Lima, E. P. (2017). Developing a big data/analytics project: A case study in the auto industry. 67th Annual Conference and Expo of the Institute of Industrial Engineers 2017; 1753-1758. Recuperado em: https://www.scopus.com/inward/record.uri?eid=2-s2.085031047139andpartnerID=40andmd5=994614a67215cffa9d37a4e0b4833dd6. Acesso em: 04 jan. 2020.

Dias, M. A. L. (2004). Extração automática de palavras-chave na língua portuguesa aplicada a dissertações e teses da área das engenharias. Dissertação de mestrado, Universidade Estadual de Campinas, Campinas, SP, Brasil.

Eck, N. J. Van; Waltman, L. (2014). Visualizing bibliometric networks. In Measuring scholarly impact (pp. 285-320). Springer.

Elsevier. (2019). What is Scopus. Recuperado em: https://www.elsevier.com/solutions/scopus. Acesso em: 22 dez. 2019.

Emmer, C.; Pfouga, A.; Stjepandic, J.; Tiringer, H. (2017). Novel approach with 3D measurement data management for industry 4.0. Advances in Transdisciplinary Engineering; 5; 906-913, Doi: https://doi.org/10.3233/978-1-61499-779-5-906

Fiesp, C. (2017). A corrida tecnológica rumo à Indústria 4.0: quem está na pole position? Recuperado em: https://www.fiesp.com.br/arquivo-download/?id=240141. Acesso em: 22 dez. 2019.

Franco, N. M. G.; de Faria, L. I. L. (2019). Colaboração científica intraorganizacional: análise de redes por coocorrência de palavras-chave. Em Questão; 25(1); 87-110.

Funk, K.; Njah, A.; Neuy, C.; Jeuk, P.-J. (2015). Industry 4.0 - Cross-cluster activities between microsystems technology; IT and virtual engineering. Smart Systems Integration 2015 - 9th International Conference and Exhibition on Integration Issues of Miniaturized Systems: MEMS; NEMS; ICS and Electronic Components; SSI 2015; 188-194. Recuperado em: https://www.scopus.com/inward/record.uri?eid=2-s2.084976264681andpartnerID=40andmd5=d65639e4f3188e3c2bcb7631517f1c28. Acesso em: 23 dez. 2019.

Geissbauer, R.; Schrauf, S.; Koch, V.; Kuge, S. (2014). Industry 4.0: Opportunities and Challenges of the Industrial Internet. PricewaterhouseCoopers.

Geng, S.; Wang, Y.; Zuo, J.; Zhou, Z.; Du, H.; Mao, G. (2017). Building life cycle assessment research: A review by bibliometric analysis. Renewable and Sustainable Energy Reviews; 76; 176-184. 
Gentner, S. (2016). Industry 4.0: Reality; future or just science fiction? How to convince today's management to invest in tomorrow's future! successful strategies for industry 4.0 and manufacturing IT. Chimia; 70(9); 628-633, Doi: https://doi.org/10.2533/chimia.2016.628

Goger, G.; Bisenberger, T. (2018). Tunnelling 4.0 - Construction-related future trends: Tunnelbau 4.0 Baubetriebliche Zukunftstrends. Geomechanik Und Tunnelbau; 11(6); 710-721, Doi: https://doi.org/10.1002/geot.201800058

Haddara, M.; Elragal, A. (2015). The Readiness of ERP Systems for the Factory of the Future. Procedia Computer Science; 64; 721-728, Doi: https://doi.org/10.1016/j.procs.2015.08.598

Hasibovic, A. C.; Tanovic, A. (2019). PRINCE2 vs Scrum in digital business transformation. 2019 42nd International Convention on Information and Communication Technology; Electronics and Microelectronics; MIPRO 2019 - Proceedings; 1514-1518, Doi: https://doi.org/10.23919/MIPRO.2019.8756716

Hendradewa, A. P. (2019). Schedule Risk Analysis by Different Phases of Construction Project Using CPM-PERT and Monte-Carlo Simulation. IOP Conference Series: Materials Science and Engineering; 528(1), Doi: https://doi.org/10.1088/1757-899X/528/1/012035

Hui, J. K. (2018). Vision for Technology Manager's Notebook. IEEE Engineering Management Review; 46(1); 10, Doi: https://doi.org/10.1109/EMR.2018.2810112

ILX, T. (2017). Things for Project Mangers to Look out for in the Fourth Industrial Revolution. Recuperado em: https://www.ilxgroup.com/usa/individual/blog/4-things-for-project-mangersto-watch. Acesso em: 3 jan. 2020.

Isaacs, D.; Astarola, A.; Diaz, J.; Arejita, B. (2017). Making factories smarter through machine learning. IIC Journal of Innovation.

Jamison, G. (2019). Industry 4.0. Recuperado em: https://www.clarehillassociates.com/blog/industry40. Acesso em: 22 dez. 2019.

Jovanović, M.; Latić, B.; Mas, A.; Mesquida, A.-L. (2015). The agile approach in industrial and software engineering project management. Journal of Applied Engineering Science; 13(4); 213-216, Doi: https://doi.org/10.5937/jaes13-9577

Júnior, W. C. da F.; WILSON, C. (2005). Análise de conteúdo. Métodos e Técnicas de Pesquisa Em Comunicação. São Paulo: Atlas; 380.

Kameiya, M.; Romeiro, M.; Kniess, C. (2017). Boas práticas em gestão de projetos: um estudo na prefeitura de Praia Grande. Revista de Administração da UFSM; 10; 870, Doi: https://doi.org/10.5902/1983465912869

Kelly, W. (2016). The Future of Digital Business: What Does This Mean for Project Teams? Recuperado em: https://www.liquidplanner.com/blog/digital-business-project-teams/. Acesso em: 22 dez. 2019.

Kerzner, H. (2018). No Project management best practices: achieving global excellence (4a ed). Hoboken: Wiley.

Kolberg, D.; Zühlke, D.; Kolberg, D.; Kolberg, D. (2015). ScienceDirect Lean Automation enabled by 
Industry Lean Automation Automation enabled enabled by by Industry, Doi: https://doi.org/10.1016/j.ifacol.2015.06.359

Kryvenchuk, Y.; Shakhovska, N.; Melnykova, N.; Holoshchuk, R. (2019). Smart Integrated Robotics System for SMEs Controlled by Internet of Things Based on Dynamic Manufacturing Processes. Advances in Intelligent Systems and Computing; Vol. 871; pp. 535-549, Doi: https://doi.org/10.1007/978-3-030-01069-0_38

Lee, J.; Bagheri, B.; Kao, H.-A. (2015). A cyber-physical systems architecture for industry 4.0-based manufacturing systems. Manufacturing Letters; 3; 18-23.

Low, S. P.; Gao, S.; Ng, E. W. L. (2019). Future-ready project and facility management graduates in Singapore for industry 4.0: Transforming mindsets and competencies. Engineering; Construction and Architectural Management, Doi: https://doi.org/10.1108/ECAM-08-20180322

Machado, R. das N. (2007). Análise cientométrica dos estudos bibliométricos publicados em periódicos da área de biblioteconomia e ciência da informação (1990-2005). Perspectivas Em Ciência Da Informação; 12(3); 2-20.

Marr, B. (2018). What is Industry 4.0? Here's A Super Easy Explanation For Anyone. Recuperado em: https://www.forbes.com/sites/bernardmarr/2018/09/02/what-is-industry-4-0-heres-a-supereasy-explanation-for-anyone/\#76933d029788. Acesso em: 22 dez. 2019.

Minitab. (2019). Suporte ao Minitab 18. Recuperado em: https://support.minitab.com/ptbr/minitab/18/getting-started/introduction/. Acesso em: 22 dez. 2019.

Miranda, S.; Sugarindra, M. (2019). Utilizing project management software in project scheduling: A case study. IOP Conference Series: Materials Science and Engineering; 528(1), Doi: https://doi.org/10.1088/1757-899X/528/1/012037

Moeuf, A.; Lamouri, S.; Pellerin, R.; Tamayo-Giraldo, S.; Tobon-Valencia, E.; Eburdy, R. (2019). Identification of critical success factors; risks and opportunities of Industry 4.0 in SMEs. International Journal of Production Research, Doi: https://doi.org/10.1080/00207543.2019.1636323

Monostori, L.; Kádár, B.; Bauernhansl, T.; Kondoh, S.; Kumara, S.; Reinhart, G.; ... Ueda, K. (2016). Cyber-physical systems in manufacturing. Cirp Annals; 65(2); 621-641.

Mozzato, A. R.; Grzybovski, D. (2011). Análise de conteúdo como técnica de análise de dados qualitativos no campo da administração: potencial e desafios. Revista de Administração Contemporânea ; Vol. 15; pp. 731-747. scielo .

Orth, M. (2018). Entendendo a Indústria 4.0. Recuperado em: https://www.deutschland.de/pt$\mathrm{br} /$ topic/economia/industria-40-tres-importantes-projetos-da-alemanha. Acesso em: $30 \mathrm{dez}$. 2019.

Pacchini, A. P. T.; Santos, J. C. D. S.; Logiudice, R.; Lucato, W. C. (2020). Indústria 4.0: barreiras para implantação na indústria brasileira. Exacta; 18(2); 278-292, Doi: https://doi.org/10.5585/exactaep.v18n2.10605

Padillo, A.; Racero, J.; Molina, J. C.; Eguía, I. (2018). PLM for education. The next generation of 
engineers. IFIP Advances in Information and Communication Technology; Vol. 540; pp. 327337, Doi: https://doi.org/10.1007/978-3-030-01614-2_30

Pereira, M. T. R.; Ferreira, F. A.; Silva; A. (2019). Information systems for industrial processes support and optimization. Proceedings of the International Conference on Industrial Engineering and Operations Management; 2019(MAR); 642-651. Recuperado em: https://www.scopus.com/inward/record.uri?eid=2-s2.085067238754 andpartner $I D=40 a n d m d 5=d 38345$ c00211fa13585a59128d2041b9. Acesso em: 22 dez. 2019.

Piengang, F. C. N.; Beauregard, Y.; Kenné, J.-P. (2017). Introduction to optimization of decision-making in coupled; dynamic and uncertain production; maintenance and product development environment. 2017 International Annual Conference of the American Society for Engineering Management; ASEM 2017. Recuperado em:

https://www.scopus.com/inward/record.uri?eid=2-s2.0-. Acesso em: $26 \mathrm{dez}$. 2019.85040091154andpartnerID=40andmd5=50d30ef5e26746ad0f2eabb219761fbd

PMI. (2018). Um Guia do Conhecimento em Gerenciamento de Projetos (6 $\left.6^{\mathrm{a}}\right)$. Newtown Square.

Qin, J.; Liu, Y.; Grosvenor, R. (2016). A Categorical Framework of Manufacturing for Industry 4.0 and Beyond. Procedia CIRP; 52; 173-178, Doi: https://doi.org/10.1016/j.procir.2016.08.005

Rajkumar, R.; Lee, I.; Sha, L.; Stankovic, J. (2010). Cyber-physical systems: the next computing revolution. Design Automation Conference; 731-736. IEEE.

Rauch, E.; Vickery, A.; Garcia, M.; Rojas, R.; Matt, D. T. (2018). Axiomatic Design based Design of a Software Prototype for Smart Shopfloor Management. MATEC Web of Conferences; 223, Doi: https://doi.org/10.1051/matecconf/201822301012

Ribeiro, J. M. (2017). O conceito da indústria 4.0 na confeção: análise e implementação. Dissertação de mestrado, Universidade do Minho, Braga, Portugal.

Rivera, C. A.; Poza, J.; Ugalde, G.; Almandoz, G. (2017). A Knowledge Based System architecture to manage and automate the electrical machine design process. Proceedings of the 2017 IEEE International Workshop of Electronics; Control; Measurement; Signals and Their Application to Mechatronics; ECMSM 2017, Doi: https://doi.org/10.1109/ECMSM.2017.7945875

Romer, R. (2016). Indústria 4.0: desafios da aplicação do "modelo alemão" no Brasil. Recuperado em: https://canaltech.com.br/negocios/industria-40-desafios-da-aplicacao-do-modelo-alemao-nobrasil-80017/. Acesso em: 30 dez. 2019.

Salehi, V. (2020). Development of an agile concept for mbse for future digital products through the entire life cycle management called Munich agile MBSE concept (MAGIC). Computer-Aided Design and Applications; 17(1); 147-166, Doi: https://doi.org/10.14733/cadaps.2020.147-166

Santos, M. H.; Emilio, D.; Pereira, A.; Henrique, T.; Silva, D. O.; Chagas, J. M. (2018). Os impactos tecnológicos no gerenciamento de projetos. Recuperado em:

https://www.aedb.br/seget/arquivos/artigos18/12126374.pdf. Acesso em: 7 dez. 2019.

Sauer, M. S.; Grosch, T.; Abele, E. (2014). Smart tool-development of an intelligent tool system. ZWF Zeitschrift Fuer Wirtschaftlichen Fabrikbetrieb; 109(7-8); 542-545, Doi:

Exacta, 20(4), p. 832-860, out./dez. 2022 
https://doi.org/10.3139/104.111185

Schelini, A.; Martens, C.; Piscopo, M. (2017). A gestão de projetos como vantagem competitiva para internacionalização de empresas brasileiras. Internext; 12; 1, Doi: https://doi.org/10.18568/1980-4865.1231-15

Schmitt, P. A. (2019). Translation 4.0-Evolution; Revolution; Innovation or Disruption? Lebende Sprachen; 64(2); 193-229.

SENAI. (2019). Benefícios da indústria 4.0. Recuperado em: https://senai40.com.br/saiba-mais/. Acesso em: 22 dez. 2019.

Stojanovic, V.; Trapp, M.; Richter, R.; Hagedorn, B.; Döllner, J. (2018). Towards the generation of digital twins for facility management based on 3D point clouds. Proceeding of the 34th Annual ARCOM Conference; ARCOM 2018; 270-279. Recuperado em: https://www.scopus.com/inward/record.uri?eid=2-s2.085054529484andpartnerID=40andmd5=362878d63e18bee90afc8761c9203163. Acesso em: 23 dez. 2019.

Team, I. (2017). 4(.0) Things for Project Mangers to Look out for in the Fourth Industrial Revolution. Recuperado em: https://www.ilxgroup.com/usa/individual/blog/4-things-for-project-mangersto-watch. Acesso em: 20 dez. 2019.

Tountopoulos, V.; Kavakli, E.; Sakellariou, R. (2018). Towards a cloud-based controller for data-driven service orchestration in smart manufacturing. Proceedings - 2018 6th International Conference on Enterprise Systems; ES 2018; 96-99, Doi: https://doi.org/10.1109/ES.2018.00022

Trusculescu, A.; Draghici, A.; Albulescu, C. T. (2015). Key Metrics and Key Drivers in the Valuation of Public Enterprise Resource Planning Companies. Procedia Computer Science; 64; 917-923, Doi: https://doi.org/10.1016/j.procs.2015.08.608

Vargas, R. V. (2005). Gerenciamento de Projetos (6a edição). Brasport.

Vergara, S. C. (2005). Métodos de Pesquisa em Administração. São Paulo: Atlas; 2005. VICO MAÑAS; Antonio. Administração de Sistemas de Informação. São Paulo: Erica.

Villar-Fidalgo, L.; Crespo Márquez, A.; González Prida, V.; De la Fuente, A.; Martínez-Galán, P.; Guillén, A. (2018). Cyber physical systems implementation for asset management improvement: $A$ framework for the transition. Safety and Reliability - Safe Societies in a Changing World Proceedings of the 28th International European Safety and Reliability Conference; ESREL 2018; 3063-3070. Recuperado em: https://www.scopus.com/inward/record.uri?eid=2-s2.085058114605 andpartner $I D=40 a n d m d 5=a d d a a 6 a 88 b 8521 d 8 d 392992 d e 63 d 2 f f 5$. Acesso em: 30 dez. 2019.

VOSviewer. (2019). Welcome to VOSviewer. Recuperado em: <https://www.vosviewer.com/>. Acesso em: 22 dez. 2019.

Wang, Y. (2015). Development of an energy management system using 'big data' and 'wireless' technologies for industrie 4.0 in brewery industry. International Journal of Simulation: Systems; Science and Technology; 16(2A); 10.1-10.4, Doi: https://doi.org/10.5013/IJSSST.a.16.2A.10 
Wolter, M. I.; Hummel, M.; Neuber-pohl, C. (2015). Industry 4.0 and the consequences for labour market and economy: Scenario calculations in line with the BIBB-IAB qualifications and occupational field projections. 12.

Yang, C. H. (2018). Development of intelligent building management system evaluation and selection for smart factory: An integrated MCDM approach. IEEE International Conference on Industrial Engineering and Engineering Management; 2017-Decem; 1505-1509, Doi: https://doi.org/10.1109/IEEM.2017.8290144

Yang, H.-L.; Chang, T.-W.; Choi, Y. (2018). Exploring the research trend of smart factory with topic modeling. Sustainability (Switzerland); 10(8), Doi: https://doi.org/10.3390/su10082779

Yin, Y.; Qin, S.-F. (2019). A smart performance measurement approach for collaborative design in Industry 4.0. Advances in Mechanical Engineering; 11(1), Doi: https://doi.org/10.1177/1687814018822570

Zaouga, W.; Rabai, L. B. A.; Alalyani, W. R. (2019). Towards an ontology based-approach for human resource management. Procedia Computer Science; 151; 417-424, Doi: https://doi.org/10.1016/j.procs.2019.04.057

Zgodavova, K.; Sutoova, A.; Cicka, M. (2019). Launching New Projects in Industry 4.0: Best Practices of Automotive Suppliers. Lecture Notes in Mechanical Engineering; pp. 183-191, Doi: https://doi.org/10.1007/978-3-030-18180-2_14

Zhong, R. Y.; Xu, X.; Klotz, E.; Newman, S. T. (2017). Intelligent Manufacturing in the Context of Industry 4. 0 : A Review. Engineering; 3(5); 616-630, Doi: https://doi.org/10.1016/J.ENG.2017.05.015 